\title{
Expression of IL-1 $\beta$, IL-6 and TNF- $\alpha$ in rats with thioacetamide-induced acute liver failure and encephalopathy: correlation with brain edema
}

\author{
Li-Qing Wang ${ }^{\mathrm{a}}$, Heng-Jun Zhou ${ }^{\mathrm{b}}$, Cai-Fei Pan ${ }^{\mathrm{a}}$, Sheng-Mei Zhu ${ }^{\mathrm{a}}$, Lin-Mei Xu ${ }^{\mathrm{a}}$ \\ ${ }^{a}$ Department of Anesthesiology; ${ }^{b}$ Department of Neurosurgery, the First Affiliated Hospital, School \\ of Medicine, Zhejiang University, Hangzhou 310003, China
}

\begin{abstract}
Background: Secondary brain edema is a serious complication of hepatic encephalopathy (HE). Recently, it has been reported that proinflammatory cytokines are involved in the pathogenesis of brain edema during HE.

Objectives: Observe the dynamic expressions of brain and plasma proinflammatory cytokines in encephalopathy rats, and evaluate the relationship between proinflammatory cytokines and brain edema.

Methods: Acute HE rats were induced by intraperitoneal injection of thioacetamide (TAA) in 24 hours intervals for two consecutive days. Then, clinical symptom and stages of hepatic encephalopathy, motor activity counts, index of liver function, and brain water content were observed. The dynamic expressions of IL-1 $\beta$, IL- 6 , and TNF- $\alpha$ in plasma and brain tissues were measured with enzyme-linked immunosorbent assay.

Results: Typical clinical performances of hepatic encephalopathy were occurred in all TAA-administrated rats. The TAA rats showed lower motor activity counts and higher the index of alanine aminotransferase, aspartate aminotransferase, total bilirubin and ammonia than those in control rats. Brain water content was significantly enhanced in TAA rats compared with the control. The expressions of IL-1 $\beta$, IL- 6 , and TNF- $\alpha$ in plasma and brain significantly increased in TAA rats. In addition, the expressions of cerebral proinflammatory cytokines were positively correlated with brain water content but negatively correlated with motor activity counts.
\end{abstract}

Conclusion: Inflammation was involved in the pathogenesis of brain edema during TAA-induced HE.

Keywords: Acute liver failure, brain edema, hepatic encephalopathy, proinflammatory cytokines, thioacetamide (TAA)

Hepatic encephalopathy (HE) is a neuropsychiatric syndrome related to both acute and chronic liver dysfunction $[1,2]$. The most prominent neurological problem is cerebral edema. Increased intracranial hypertension, resulting predominantly in brain herniation and extremely high mortality rates $[3,4]$.

The precise pathophysiologic mechanisms responsible for cerebral edema formation induced by HE have not been understood fully. Ammonia may be important in the pathogenesis of HE and brain edema [5]. However, increasing evidence suggests

Correspondence to: Prof. Sheng-Mei Zhu, Department of Anesthesiology, the First Affiliated Hospital, School of Medicine, Zhejiang University, Hangzhou 310003, PR China. E-mail:smzhu20088@yahoo.com.cn that inflammation plays a significant role in the molecular pathogenesis of HE and brain edema $[6,7]$. For example, the presence of systemic inflammation is correlated with a more rapid progression of encephalopathy and brain edema in ALF patients [8]. Recently, Chu et al [9] demonstrated that a significant correlation between plasma levels of tumor necrosis factor (TNF)- $\alpha$ and severity of HE in rats with thoacetamide-induced fulminant hepatic failure. Indeed, circulating proinflammatory cytokines correlate with encephalopathy and brain edema in acute liver failure (ALF) and ammonia. However, no conclusive evidence has been obtained for increased brain proinflammatory cytokines production in HE.

In this study, we observed the expression of plasma and brain proinflammatory cytokines and assessed its possible relationship between cerebral 
inflammation and brain edema in thioacetamide (TAA) "administrated rats with acute hepatic failure and HE. In particular, stages of HE, motor activity counts, biochemical analyses, and brain water content were measured as a function of encephalopathy and brain edema severity. Expression of the plasma and brain proinflammatory cytokines (IL-1 $\beta$, IL- 6 , and TNF- $\alpha$ ) were measured using enzyme-linked immunosorbent assay.

\section{Materials and methods}

\section{Animal model and experimental design}

The experimental protocols were approved by the Medical Faculty Ethics Committee of Zhejiang University. All experimental procedures were performed in accordance with the Guide for the Care and Use of Experimental Animals.

Male Sprague-Dawley rats (220-250g) obtained from the Experimental Animal Center in the Zhejiang Academy of Medical Sciences. The rats were caged at $24^{\circ} \mathrm{C}$, with a 12 -hour light-dark cycle and free access to food and water before experimentation. Acute hepatic failure and encephalopathy was induced by intraperitoneal injection of TAA $(350 \mathrm{mg} / \mathrm{kg}$ in normal saline, TCI Co, Tokyo, Japan) at 24-hours interval for two consecutive days $(\mathrm{n}=24)$ [10-12]. Rats administrated with normal saline were classified as controls $(n=24)$. Each group was further divided into three subgroups by the time of decapitation after the second TAA-administration, 24 hours $(\mathrm{n}=8), 48$ hours $(n=8)$ and 60 hours $(n=8)$. All the rats were given a supportive therapy that consisted of $5 \%$ glucose and $0.45 \%$ saline mixed with $20 \mathrm{mEql}$ of potassium chloride $(25 \mathrm{~mL} / \mathrm{kg})[13,14]$.

Clinical symptoms were observed during the study period. Stage of hepatic encephalopathy and motor activity counts were assessed at 24 hours, 48 hours, and 60 hours after the second administration of TAA. Heparinized blood samples were obtained afterwards from abdominal aorta for biochemical determination including alanine aminotransferase (ALT), aspartate aminotransferase (AST), total bilirubin (TBIL), and ammonia. The rats were sacrificed at different timepoints $(24,48$, and 60 hours after the second TAAadministration) by decapitation in deep anesthesia. Brains were rapidly removed and were dissected out and kept at $-80 \quad \mathrm{C}$ for further studies. The experimental procedure and a flow chart are shown in Figure 1.

\section{Assessment of hepatic encephalopathy (HE)}

Clinical symptom of rats and stages of HE in the different groups were assessed at different time-periods $(24,48$, and 60 hours $)$ following administration of the second dose of TAA. The stage of encephalopathy was determined using a clinical and neuro-behavioral scale as follows:

Stage 1: lethargy,

Stage 2: mild ataxia,

Stage 3: lack of spontaneous movement and loss of righting reflex, but still responsive,

Stage 4: coma and lack of response to pain [10].

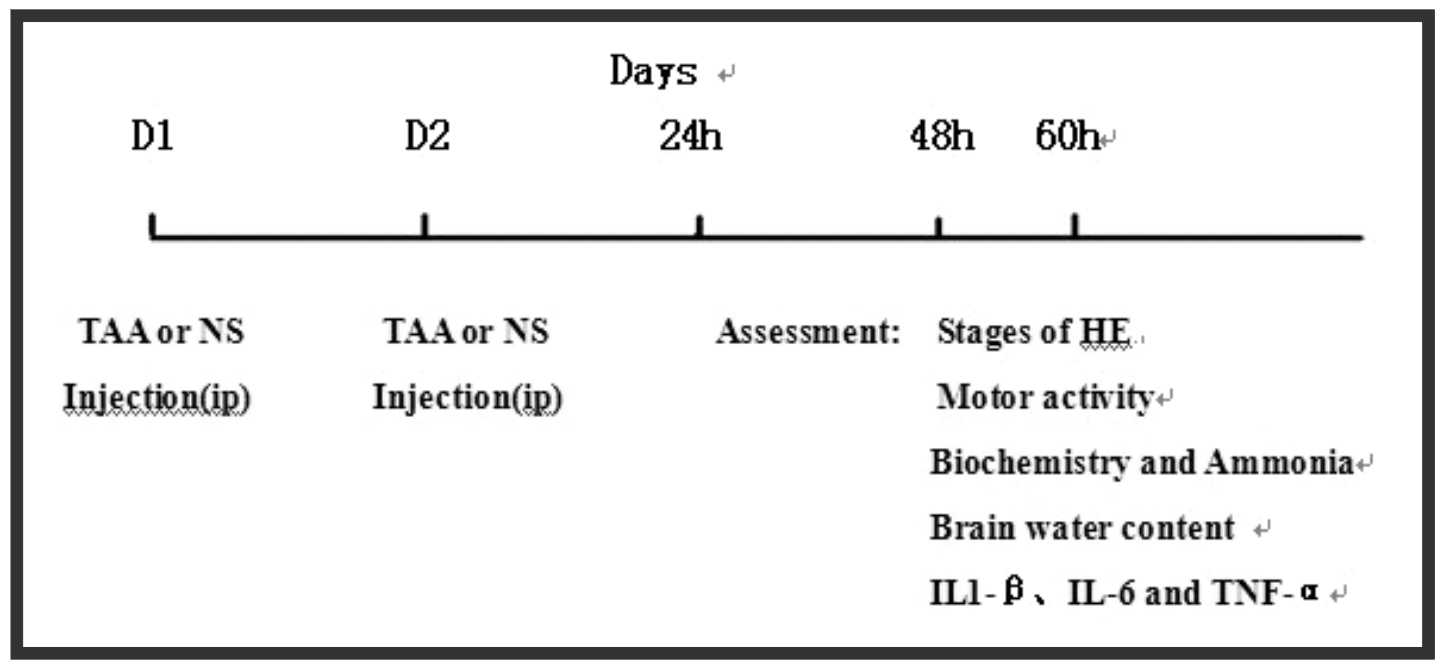

Figure 1. The entire experimental procedure. D1=the first day for TAA or NS injection, D2= the second day for TAA or NS injection, 24h: 24 hours after the second TAA or NS injection, 48h: 48 hours after the second TAA or NS injection, 60h:60 hours after the second TAA or NS injection. 
The clinical symptom of rats and survival rates in the different groups was recorded on a daily basis following administration of the first dose of TAA.

\section{Measurement of motor activities}

Motor activities were measured at different timepoints $(24,48$, and 60 hours) following administration of the second dose of TAA/NS. Motor activities were determined in the open field $(100 \times 100 \times 40 \mathrm{~cm})$ divided into 25 small squares equally $(20 \times 20 \mathrm{~cm})$ [15]. All studies were performed under standardized conditions in the dark room. Two rats from the model and the control group were observed simultaneously. Motor activity counts were recorded by counting the small squares rats crossed-over. Results were presented as total movements in 20 minutes to reflect motor activity counts of the study rats.

\section{Biochemical analyses and ammonia}

At different time-periods (24, 48, and 60 hours) following administration of the second dose of TAA/ NS, a laparotomy was performed under anesthesia with chloral hydrate $(400 \mathrm{mg} / \mathrm{kg}$, ip). The abdomen was opened wide and blood samples $(4 \mathrm{~mL})$ were taken from the abdominal aorta for biochemical determinations including aspartate transaminase (AST), alanine transaminase (ALT), total biluribin (TBIL), and ammonia. These samples were kept at room temperature for 30 minutes and centrifuged at $3000 \mathrm{rpm}$ for 10 minutes. Plasma samples obtained in this way were aliquoted and stored in a freezer $(-80 \quad$ C) for use in biochemical analyses.

Plasma biochemistry tests (ALT, AST, and TBIL) and ammonia were analyzed using an automatic biochemistry analyzer (Hitachi 7600-110, Tokyo, Japan) in the routine biochemistry laboratories of the First Affiliated Hospital of College of Medicine, Zhejiang University. AST and ALT levels are expressed as U/L, TBIL and ammonia levels as $\mu \mathrm{mol} / \mathrm{L}$.

\section{Measurement of brain water content}

To quantify cerebral edema, we used the wet and dry weight method. Animals were decapitated, and the brains were immediately removed. Half of the brain was weighed before and after 48 hours incubation in a $100^{\circ} \mathrm{C}$ oven. Water content of the brain samples were measured by the wet and dry weight method as follows [16]:
Water content $(\%)=[$ Wet weight $(\mathrm{mg})$ - Dry weight $(\mathrm{mg})]$ / Wet weight (mg) x 100\%.

\section{Enzyme-linked immunosorbent assay}

Cortical brain tissues were collected at different time-points (24, 48, and 60 hours). Tissues were homogenized in two volumes of $0.01 \mathrm{~mol} / \mathrm{L}$ phosphatebuffered saline (PBS) containing 0.05\% Tween-20. After homogenization and centrifugation at $10,000 \mathrm{~g}$ at $4^{\circ} \mathrm{C}$ for 20 minutes, the resultant supernatant was collected and stored at $-80^{\circ} \mathrm{C}$ until use. Plasma samples were collected and aliquoted as those for use in biochemical analyses and stored in a freezer $\left(-80^{\circ} \mathrm{C}\right)$ for further studies.

IL-1 $\beta$, IL- 6 and TNF- $\alpha$ concentrations were measured in brain and plasma samples using a quantitative sandwich enzyme-linked immunosorbent assay (ELISA) with monoclonal antibodies specific for rat IL-1, IL-6 and TNF- $\alpha$ (R\&D Systems, Minneapolis, USA). The micorplates were read at 450 $\mathrm{nm}$. The absorbances were converted to $\mathrm{pg} / \mathrm{ml}$ using standard curves. The lowest detection limits were IL$6(60 \mathrm{pg} / \mathrm{mL}), \mathrm{IL}-1 \beta(30 \mathrm{pg} / \mathrm{mL}), \mathrm{TNF}-\alpha(30 \mathrm{pg} / \mathrm{mL})$. All results were expressed in $\mathrm{pg} / \mathrm{mL}$.

\section{Statistical analysis}

All data in this study were expressed as mean \pm standard deviation (SD) value. Statistical analysis was performed using independent $\mathrm{T}$ test and bivariate correlation with Pearson's test, accordingly by SPSS 16.0 software. Statistical significance was set at a value of $\mathrm{p}<0.05$.

\section{Results}

\section{Hepatic encephalopathy and symptoms}

The control group acted freely, responded keenly, and gained significantly weight. All TAA-administrated rats $(n=24)$ presented typical hepatic encephalopathy symptoms including weight obviously lost, lethargy, mild ataxia, lack of spontaneous movement. The changes of body weight in the control and model groups were shown in Figure 2. Out of the original 48 animals, two died before the following examination:

zero in the control group and two in the 60 hours group.

Table 1 shows stages of HE for the study groups at different time-periods $(24,48$, and 60 hours) following administration of the second dose of TAA. No evidence of encephalopathy was detected in the control group. TAA-administrated rats developed encephalopathy progressively over the time course. 


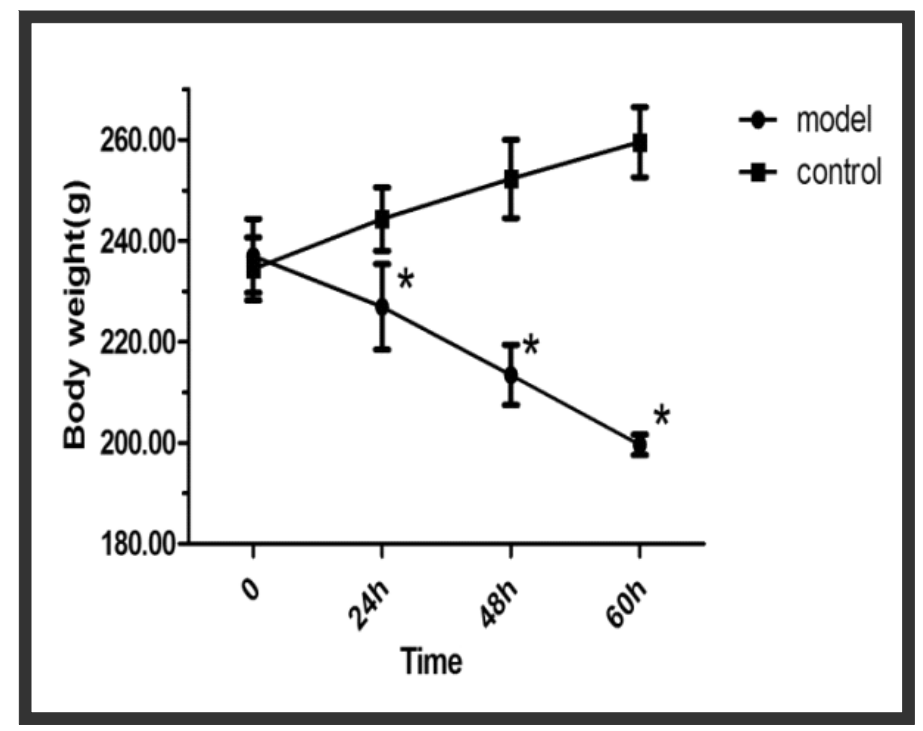

Figure 2. Mean \pm SD values of body weight by groups. Body weight all significantly decreased in the model group. $* \mathrm{p}<0.05$ are significantly different as compared to the control values.

Table 1. Stages of hepatic encephalopathy (HE) by groups.

\begin{tabular}{|c|c|c|c|c|c|c|}
\hline & \multicolumn{6}{|c|}{ Stages of $\mathrm{HE}$} \\
\hline & Number & $\mathbf{0}$ & I & II & III & IV \\
\hline \multicolumn{7}{|l|}{24 hours } \\
\hline Control & 8 & 8 & 0 & 0 & 0 & 0 \\
\hline Model & 8 & 0 & 7 & 1 & 0 & 0 \\
\hline \multicolumn{7}{|l|}{48 hours } \\
\hline Control & 8 & 8 & 0 & 0 & 0 & 0 \\
\hline Model & 8 & 0 & 3 & 4 & 1 & 0 \\
\hline \multicolumn{7}{|l|}{60 hours } \\
\hline Control & 8 & 8 & 0 & 0 & 0 & 0 \\
\hline Model & 6 & 0 & 1 & 1 & 4 & 0 \\
\hline
\end{tabular}

\section{Motor activities}

Motor activities of the control and model groups are shown in Figure 3. The model groups showed significantly lower motor activity counts than their corresponding control groups in total movements $(p<0.05)$. Behavioral function and encephalopathy of TAA-administrated rats aggravated progressively over the time course.

\section{Plasma ALT, AST, TBIL and ammonia}

Table 2 shows mean SD values for AST, ALT, TBIL and Ammonia levels at different time-points $(24,48$, and 60 hours) in the model and control groups. The model groups showed significantly higher plasma levels of ALT, AST, TBIL and Ammonia than those of their corresponding control groups $(\mathrm{p}<0.05)$. TAAadministrated rats developed hepatic injury progressively over the time-course.

\section{Brain water content}

The brain water content was only mildly elevated in the 24 hours group but there was no significant difference between the model and control rats. At 48 and 60 hours, TAA-administrated rats showed a significantly higher brain water content compared with the corresponding control rats in Figure $4(p<0.05)$. Brain water content of TAA-administrated rats developed a significant deterioration over the timecourse. 


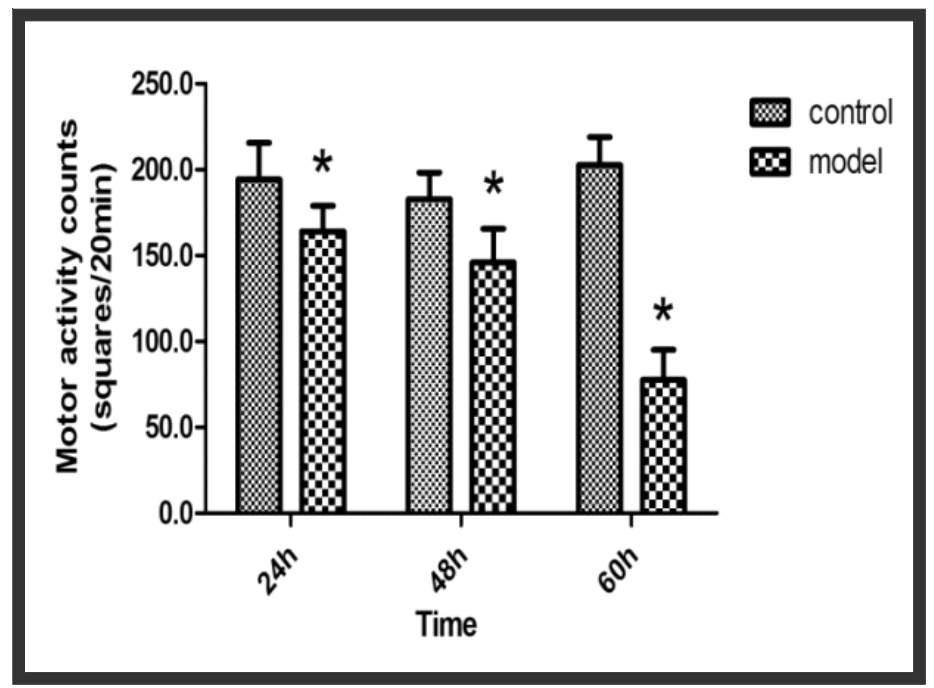

Figure 3. Mean \pm SD values of motor activity counts by groups. Total motor activity counts in the open field were significantly decreased in TAA-administrated rats. ${ }^{*} \mathrm{p}<0.05$ are significantly different compared to the control values.

Table 2. Mean \pm SD values of ALT, AST, TBIL, and Ammonia levels by groups. *p $<0.05$ are significantly different as compared to the control values.

\begin{tabular}{cllll}
\hline & $\begin{array}{l}\text { ALT } \\
(\mathbf{U} / \mathbf{L})\end{array}$ & $\begin{array}{l}\text { AST } \\
(\mathbf{U} / \mathbf{L})\end{array}$ & $\begin{array}{l}\text { Tbil } \\
(\mu \mathrm{mol} / \mathbf{L})\end{array}$ & $\begin{array}{l}\text { Ammonia } \\
(\mu \mathrm{mol} / \mathbf{L})\end{array}$ \\
\hline $\mathbf{2 4}$ hours & & & & \\
Control & $42.70 \pm 9.18$ & $101.37 \pm 38.19$ & $4.03 \pm 0.65$ & $48.00 \pm 20.60$ \\
$\quad \begin{array}{l}\text { Model } \\
\mathbf{4 8} \text { hours }\end{array}$ & $64.69 \pm 14.76^{*}$ & $192.25 \pm 40.08^{*}$ & $4.23 \pm 0.96$ & $116.2 \pm 30.27^{*}$ \\
Control & $32.67 \pm 8.14$ & $89.44 \pm 36.49$ & $3.41 \pm 0.64$ & $56.53 \pm 31.13$ \\
$\quad \begin{array}{l}\text { Model } \\
\mathbf{6 0} \text { hours } \\
\text { Control }\end{array}$ & $209.9 \pm 48.27^{*}$ & $329.4 \pm 137.51^{*}$ & $4.74 \pm 0.79^{*}$ & $148.5 \pm 56.1^{*}$ \\
Model & $40.59 \pm 6.72$ & $95.12 \pm 29.14$ & $3.08 \pm 0.63$ & $47.89 \pm 22.14$ \\
& $308.10 \pm 67.37^{*}$ & $680.1 \pm 180.94^{*}$ & $10.09 \pm 1.29^{*}$ & $202.61 \pm 61.3^{*}$ \\
\hline
\end{tabular}

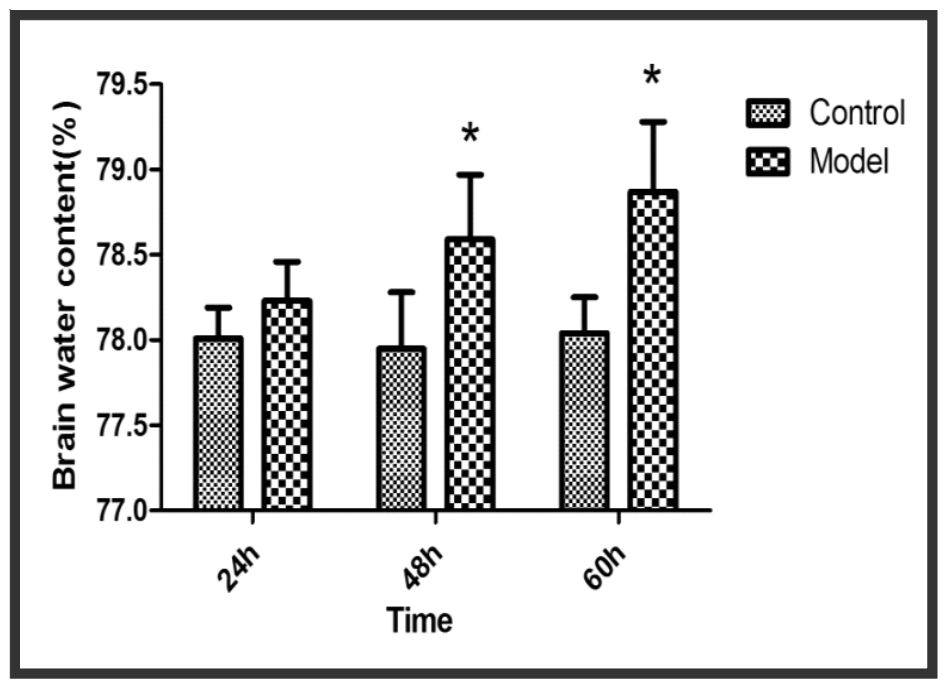

Figure 4. Mean $\pm \mathrm{SD}$ values of brain water content by groups. $* p<0.05$ are significantly different compared to the control values. 


\section{IL-1, IL-6, and TNF-a concentrations}

The brain IL-1 $\beta$, IL- 6 and TNF- $\alpha$ concentra- tions can be seen in Figure 5. The plasma concentrations show in Figure 6.

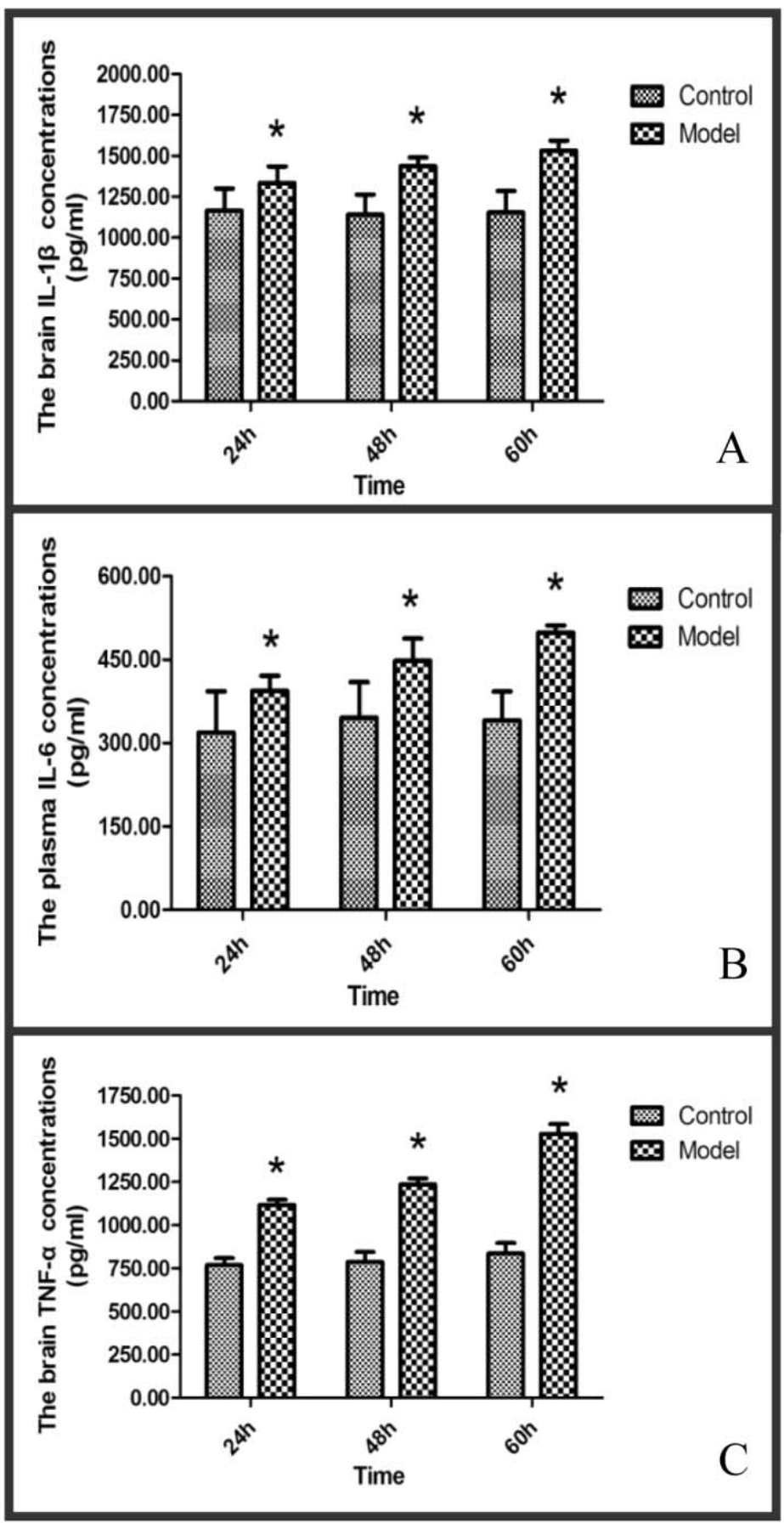

Figure 5. Mean SD values of the brain concentrations of IL-1 $\beta$ (A), IL-6 (B), and TNF- $\alpha$ (C). TAA-administrated rats showed significantly higher brain levels of IL- $1 \beta$, IL-6, and TNF- $\alpha$ than the corresponding control. * $p<0.05$ are significantly different as compared to the control values. 


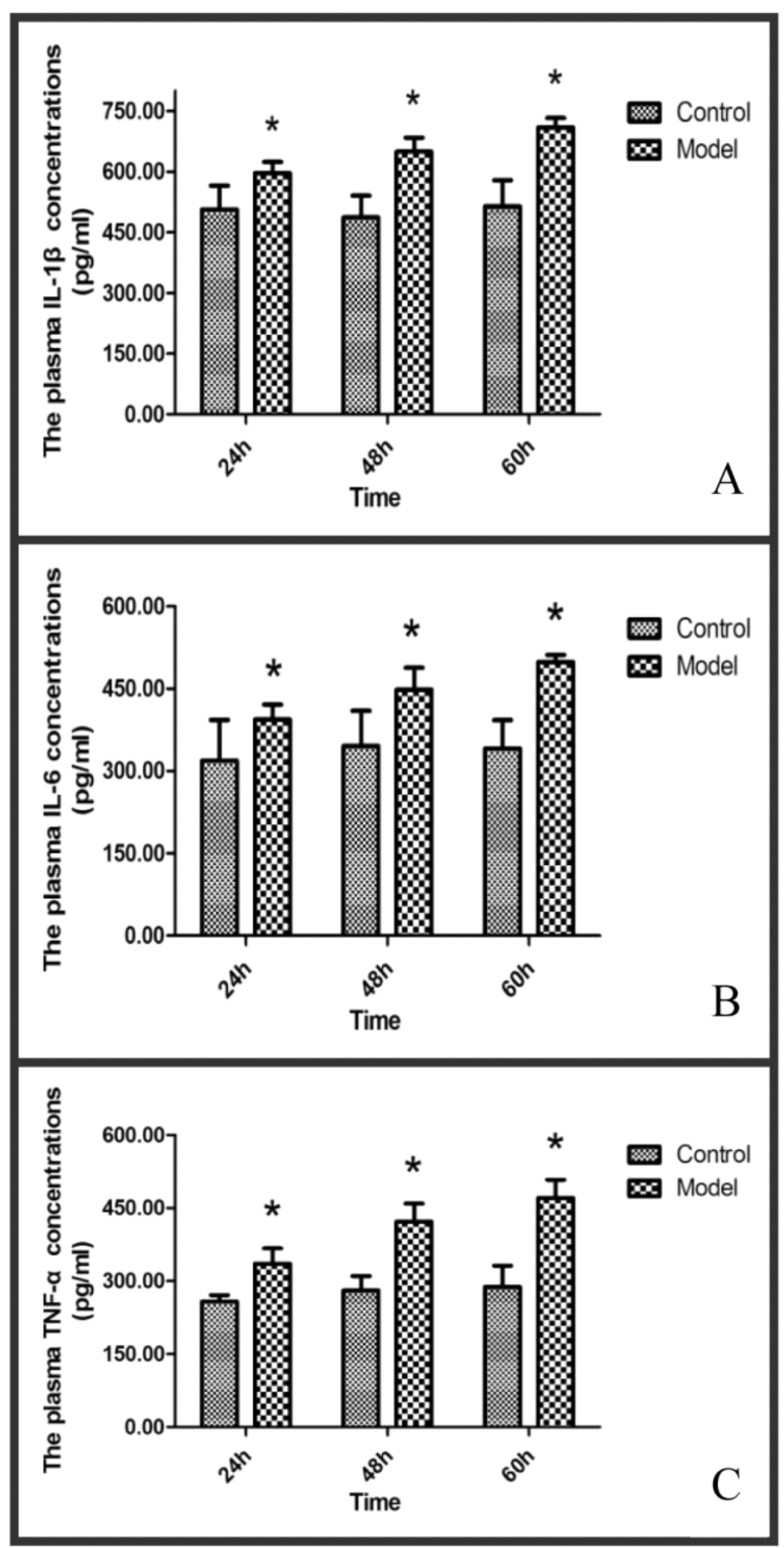

Figure 6. Mean \pm SD values of the plasma concentrations of IL-1 $\beta$ (A) IL-6 (B) and TNF- (C) TAA-administrated rats showed significantly higher plasma levels of IL-1 $\beta$, IL-6, and TNF- $\alpha$ than the corresponding control. *p $<0.05$ are significantly different as compared to the control values. 
TAA-administrated rats showed significantly higher levels of the brain and plasma IL-1 $\beta$ (7A), IL6 (7B), and TNF- $\alpha$ (7C) than those of their corresponding control groups $(\mathrm{p}<0.05)$. IL-1 $\beta(\mathbf{8 A})$, IL-6 (8B) and TNF- $\alpha(\mathbf{8 C})$ concentration increased gradually from the 24 hours after the second administration of TAA in TAA-administrated rats and reached a maximal level at the 60 hours. Apparently, from the data of Figure 5 and $\mathbf{6}$, higher levels of the brain IL- $1 \beta$, IL- 6 , and TNF- $\alpha$ were significantly observed in TAA-administrated rats, compared to the plasma.

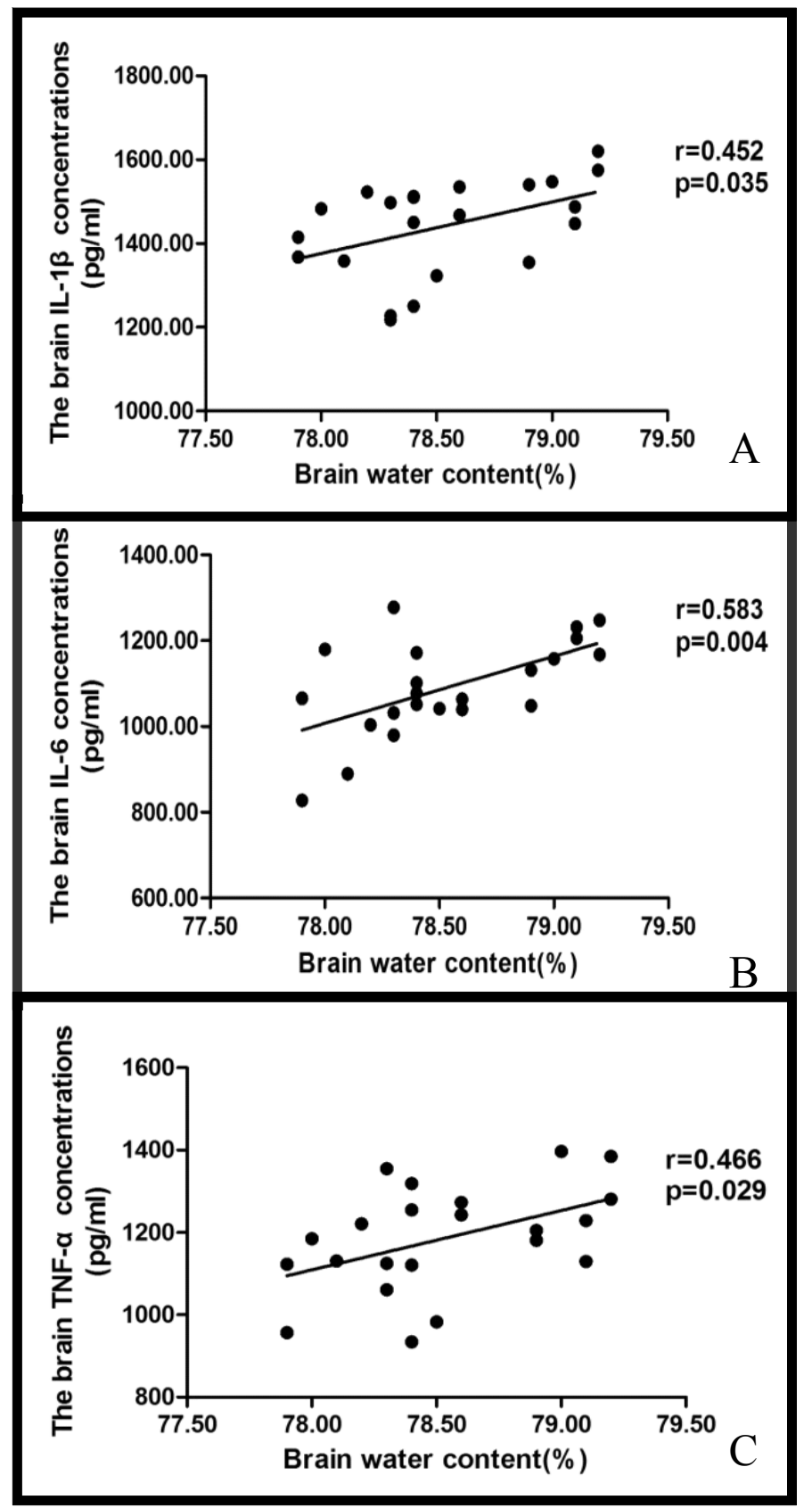

Figure 7. Correlations between the brain levels of IL-1 $\beta$ (A), IL-6 (B), and TNF- $\alpha$ (C) and brain water content in TAAadministrated rats. The significant positive correlations were noted. 
Correlations between proinflammatory cytokines (IL-1 $\beta, I L-6$ and TNF- $\alpha$ ) concentration, brain water content and $\mathrm{HE}$

Figure 7 and 8 show correlations between the brain levels of IL- $1 \beta$, IL- 6 , and TNF- $\alpha$ and brain water content, and between the levels of IL-1 $\beta$, IL-6, and TNF- $\alpha$ and HE, in TAA-administrated rats.

Interestingly, brain concentrations of IL-1 $\beta$, IL-6, and TNF- $\alpha$ were significantly positive correlated with brain water content in TAA-administrated rats, as shown in Figure 7. This indicates that more severe brain inflammation was associated with aggravated brain edema. In addition, brain concentrations of IL-1 $\beta$, IL- 6 , and TNF- $\alpha$ were negatively correlated with total motor activity counts (Figure 8). This suggests that aggregated HE and brain edema are accompanied by IL- $1 \beta$, IL- 6 , and TNF- $\alpha$ protein upregulation.

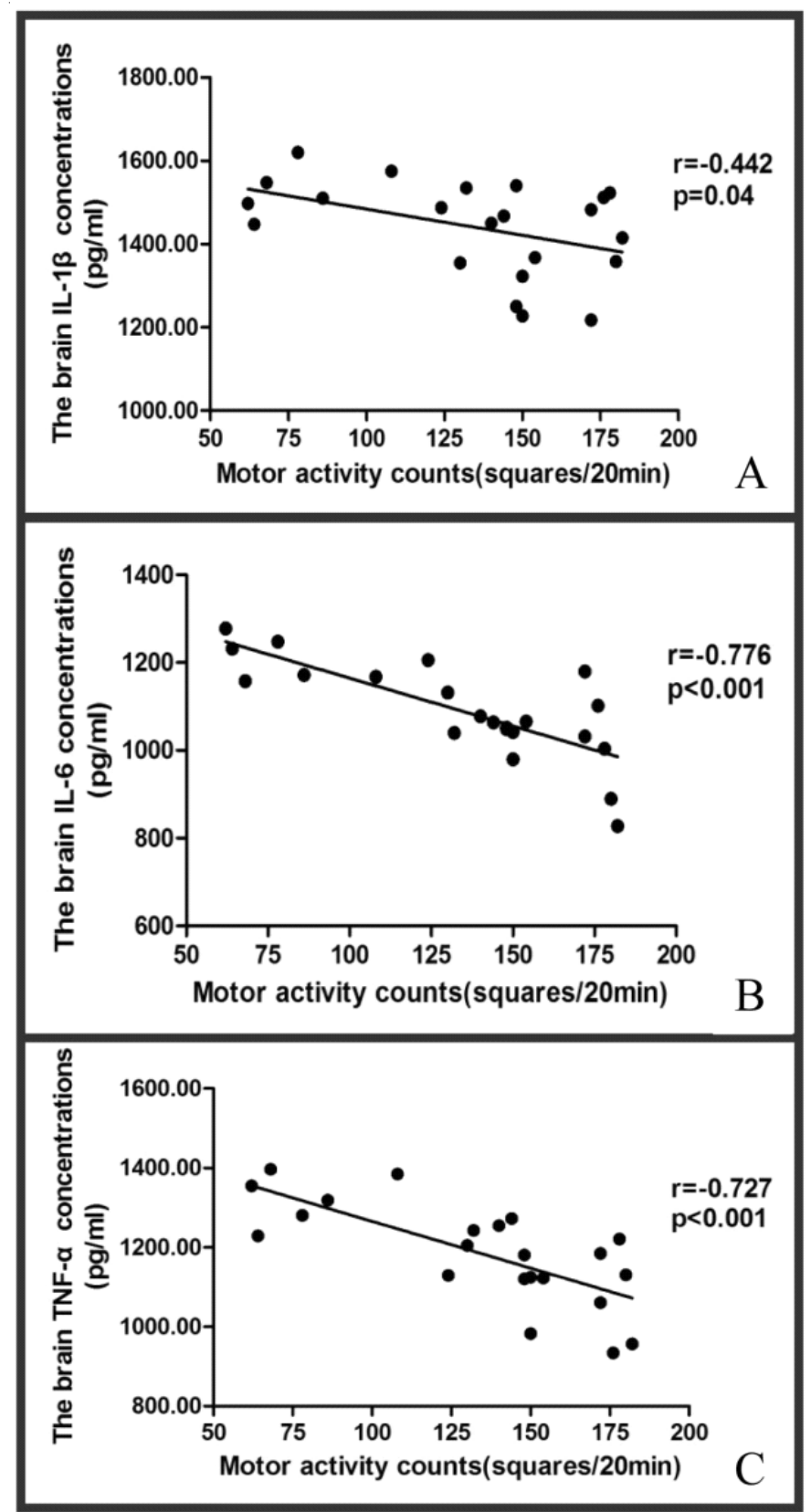

Figure 8. Correlations between the levels of IL-1 $\beta$ (A), IL-6 (B), and TNF- $\alpha$ (C) and HE in TAAadministrated rats. The significantly negative correlations were shown. 


\section{Discussion}

The experimental rat model resembling acute hepatic failure and HE induced by TAA was extensively proved to be a validated and satisfactory model of HE [10-12, 17, 18]. In the present study, we developed TAA-induced acute hepatic failure in rats, and showed that all the rats presented typical hepatic encephalopathy symptoms (weight lost, lethargy, mild ataxia, and lack of spontaneous movement). The body weight and total motor activity counts significantly decreased compared with the control, as shown in Figures 2 and 3. In addition, rats-administrated with TAA induced severe liver damage associated with elevated levels of liver enzymes and blood ammonia (see Table 2).

Our study showed that increase in expression of proinflammatory cytokines (IL-1 $\beta$, IL-6, and TNF$\alpha$ ) was observed in rats with TAA-induced acute hepatic failure and encephalopathy. The brain and plasma proinflammatory cytokines (IL-1 $\beta$, IL-6, and TNF- $\alpha$ ) concentrations were significantly elevated in TAA group compared with the control 9, as shown in Figures 5 to 8. In addition, they showed significantly negative correlations with motor activity counts. This indicates that inflammation developed aggressively accompanied with aggravated HE. Interestingly, this finding is compatible with the hypothesis that severer HE is associated with more severe cerebral inflammation. According to $\mathrm{Chu}$ et al [19], plasma levels of TNF- $\alpha$ in rats with thioacetamide-induced fulminant hepatic failure should be significantly associated with more blunted motor activity. Furthermore, HE developed more frequently in rats with higher plasma levels of TNF- $\alpha$. Another recent study using a similar experimental model has reported a correlation between plasma levels of TNF- $\alpha$ and the severity of HE [9]. These findings add to evidences that inflammation may play a role in the pathogenesis of HE associated with acute liver failure.

ALF-associated HE is characterized by cerebral edema that elevates intracranial pressure. In acute HE, astrocytes swell and develop cytotoxic brain edema [20]. As seen in Figure 4, significantly higher brain water content increased in the 48 and 60 hours TAA group compared with the corresponding control. The brain water content of 24 hours TAA group was elevated but not significantly changed, compared with the control. This indicates that rats did not show brain edema in the earlier period of HE. However, our result showed that proinflammatory cytokines production occurred prior to the appearance of brain edema. The correlation analysis of proinflammatory cytokines and brain water content (Figure 7) showed that the brain concentrations of IL-1 $\beta$, IL- 6 , and TNF- $\alpha$ were positively correlated with brain water content in TAAadministrated rats. This indicates that a progressive brain edema was accompanied by significant increases in expression of brain proinflammatory cytokines IL-1 $\beta$, IL- 6 , and TNF- $\alpha$. Excessive proinflammatory factors formation in the initial stage of HE may lead to aggravation of brain edema, which may participate in the development of HE and brain edema. These findings strengthen the notion that cerebral accumulation of proinflammatory cytokines are implicated in the pathogenesis of brain edema in HE.

In this study, we found enhanced brain and plasma IL-1 $\beta$, IL- 6 , and TNF- $\alpha$ concentrations in rats with TAA-induced fulminant hepatic failure and HE. However, we also found the brain proinflammatory cytokines (IL-1 $1 \beta$, IL- 6 and TNF- $\alpha$ ) concentration was much higher than the plasma in TAA-administrated rats (see Figures 5 and 6). Our findings suggest that increases in proinflammatory cytokines and brain edema in rats TAA-induced HE is likely to be two evolutive-step processes as follows. The first is that in ALF, the necrotic liver may release circulating proinflammatory cytokines. The second being brain inflammation, possibly due to astrocytes that produces cytokines. Astrocytes are the key cells involved in the pathogenesis of HE and has extensive repertoire to modulate inflammatory responses [4]. The possible mechanism that circulating proinflammatory cytokines may stimulate the activation of astrocytes in HE through circumventricular organs that lack a bloodbrain barrier to secrete cytokines [21]. As a result, the circulating inflammatory process would cause a worsening of brain inflammation and a release like a chute of proinflammatory cytokines. Therefore, cerebral inflammation actually may play a more significant role in the pathogenesis of brain edema in hepatic encephalopathy.

In conclusion, inflammation might be associated with more severe HE and brain edema, particularly cerebral proinflammatory cytokines. Experimental therapeutic studies with anti-inflammatory agents with the capacity to brain proinflammatory cytokines production in HE will be warranted. 


\section{Acknowledgment}

This research was supported by the Medical Science Research Foundation of Health Bureau of Zhejiang Province of China (2007A057). The authors have no conflict of interest to declare.

\section{References:}

1. Aggarwal S, Kramer D, Yonas H, Obrist W, Kang Y, Martin M, et al. Cerebral hemodynamic and metabolic changes in fulminant hepatic failure: a retrospective study. Hepatology. 1994; 19:80-7.

2. Hazell AS, Butterworth RF. Hepatic encephalopathy: An update of pathophysiologic mechanisms. Proc Soc Exp Biol Med. 1999; 222:99-112.

3. Lewis M, Howdle PD. The neurology of liver failure. QJM. 2003; 96:623-33.

4. Shawcross D, Jalan R. The pathophysiologic basis of hepatic encephalopathy: central role for ammonia and inflammation. Cell Mol Life Sci. 2005; 62:2295-304.

5. Cooper AJ, Plum F. Biochemistry and physiology of brain ammonia. Physiol Rev. 1987; 67:440-519.

6. Vaquero J, Polson J, Chung C, Helenowski I, Schiodt $\mathrm{FV}$, Reisch J, et al. Infection and the progression of hepatic encephalopathy in acute liver failure. Gastroenterology. 2003; 125:755-64.

7. Shawcross DL, Davies NA, Williams R, Jalan R. Systemic inflammatory response exacerbates the neuropsychological effects of induced hyperammonemia in cirrhosis. J Hepatol. 2004; 40: 247-54.

8. Rolando N, Wade J, Davalos M, Wendon J, PhilpottHoward J, Williams R. The systemic inflammatory response syndrome in acute liver failure. Hepatology. 2000; 32:734-9.

9. Chu CJ, Wang SS, Lee FY, Chang FY, Lin HC, Hou MC, et al. Detrimental effects of nitric oxide inhibition on hepatic encephalopathy in rats with thioacetamideinduced fulminant hepatic failure. Eur J Clin Invest. 2001;31:156-63.

10. Zimmermann C, Ferenci P, Pifl C, Yurdaydin C, Ebner J, Lassmann $\mathrm{H}$, et al. Hepatic encephalopathy in thioacetamide-induced acute liver failure in rats: characterization of an improved model and study of amino acid-ergic neurotransmission. Hepatology. 1989; 9:594-601.
11. Reddy PV, Murthy C, Reddanna P. Fulminant hepatic failure induced oxidative stress in nonsynaptic mitochondria of cerebral cortex in rats. Neurosci Lett. 2004; 368:15-20.

12. Swapna I, Kumar KV, Reddy PV, Murthy C, Reddanna P, Senthilkumaran B. Phospholipid and cholesterol alterations accompany structural disarray in myelin membrane of rats with hepatic encephalopathy induced by thioacetamide. Neurochem Int. 2006; 49:238-44.

13. Norton NS, McConnell JR, Rodriguez-Sierra JF. Behavioral and physiological sex differences observed in an animal model of fulminant hepatic encephalopathy in the rat. Physiol Behav. 1997; 62:1113-24.

14. Panikashvili D, Simeonidou C, Ben-Shabat S, Hanus L, Breuer A, Mechoulam R, et al. An endogenous cannabinoid (2-AG) is neuroprotective after brain injury. Nature. 2001; 413:527-31.

15. Fride E, Mechoulam R. Pharmacological activity of the cannabinoid receptor agonist, anandamide, a brain constituent. Eur J Pharmacol. 1993; 231:313-4.

16. Zhan C, Yang J. Protective effects of isoliquiritigenin in transient middle cerebral artery occlusion-induced focal cerebral ischemia in rats. Pharmacol Res. 2006; 53:303-9.

17. Schnur J, Olah J, Szepesi A, Nagy P, Thorgeirsson SS. Thioacetamide-induced hepatic fibrosis in transforming growth factor beta-1 transgenic mice. Eur J Gastroenterol Hepatol. 2004; 16:127-33.

18. Honda H, Ikejima K, Hirose M, Yoshikawa M, Lang T, Enomoto N, et al. Leptin is required for fibrogenic responses induced by thioacetamide in the murine liver. Hepatology. 2002;36:12-21.

19. Chu CJ, Chen CT, Wang SS, Lee FY, Chang FY, Lin HC, et al. Hepatic encephalopathy in rats with thioacetamide-induced fulminant hepatic failure: role of endotoxin and tumor necrosis factor-alpha. Zhonghua Yi Xue Za Zhi (Taipei). 2001; 64:321-30.

20. Kato M, Hughes RD, Keays RT, Williams R. Electron microscopic study of brain capillaries in cerebral edema from fulminant hepatic failure. Hepatology. 1992; 15:1060-6.

21. Maness LM, Kastin AJ, Farrell CL, Banks WA. Fate of leptin after intracerebroventricular injection into the mouse brain. Endocrinology. 1998; 139:4556-62. 